\title{
Effects of Impurity Vertex Correction on NMR Coherence Peak in S-Wave Superconductors
}

\author{
Han-Yong Choi \\ Department of Physics, Sung Kyun Kwan University, Suwon 440-746, Korea \\ E. J. Mele \\ Department of Physics, University of Pennsylvania, Philadelphia, PA 19104, USA
}

(Feb. 10, 1995)

\begin{abstract}
We study the effects of non-magnetic impurity vertex correction on nuclear spin-lattice relaxation rate $1 / T_{1}$ of conventional s-wave superconductors within the Eliashberg formalism. We obtain, with a self-consistent $t$-matrix treatment of impurity scatterings, the expressions for impurity vertex function and nuclear spin-lattice relaxation rate. The $1 / T_{1}$ is evaluated with a simple approximation on angular average, and found to agree in clean limit with the previous result that $1 /\left(T_{1} T\right)$ remains unrenormalized under the impurity vertex correction. As dirtiness is increased, on the other hand, the coherence peak in $1 /\left(T_{1} T\right)$ is found to increase due to the impurity vertex correction. This result is discussed in connection with the experimental observations on conventional superconductors.
\end{abstract}

Typeset using REVTEX 
Nuclear magnetic resonance (NMR) relaxation rate $T_{1}^{-1}$ measurements have been used extensively to study both normal and superconducting properties of various materials [1]. In simple metals, the conduction electrons provide the dominant relaxation channel for polarized nuclear spins, which results in the well-known Korringa law $T_{1}^{-1} \propto T$. Below the transition temperature, $T_{c}$, the superconducting gap opens and, consequently, density of states (DOS) increases. The former and the latter, respectively, suppresses and increases the relaxation rate, so that the relaxation rate is determined by the competition between the two: At temperatures immediately below $T_{c}$, the DOS effect dominates and there is an increase of $\left(T_{1} T\right)^{-1}$ relative to the normal state Korringa value at $T=T_{c}$. At the low temperature regime, on the other hand, the effect of gap opening takes over and the relaxation rate falls off exponentially or algebraically depending on the symmetry of the superconducting gap. This peak as a function of temperature is known as coherence peak, or Hebel-Slichter peak in NMR, whose observation was very important in establishing the Bardeen-Cooper-Schrieffer (BCS) theory [2] [4].

There are several factors that affect the NMR coherence peak of $\left(T_{1} T\right)^{-1}$ [5]. The suppression of NMR coherence peak may be attributed to (a) gap anisotropy [5]/non s-wave pairing of superconducting phase [6,7], (b) strong coupling damping [8 10], (c) paramagnetic impurities in samples [5,11], and/or (d) strong Coulomb interaction such as paramagnon/antiparamagnon effects [12,13]. The non-magnetic impurity scatterings, on the other hand, have no influence on $\left(T_{1} T\right)^{-1}$ [1, 14, 15, other than the smearing of gap anisotropy. This is because (a) there exists a simple scaling relation between the renormalization function of pure $(Z(\omega))$ and impure $(\widetilde{Z}(\omega))$ superconductors and (b) in the expression for $\left(T_{1} T\right)^{-1}$, the numerator and denominator are of the same powers in $\widetilde{Z}(\omega)$, as will be discussed in more detail later. Experimentally, it was observed in a series of NMR experiments on Al- 16] and In-based alloys [17 that the coherence peak in $\left(T_{1} T\right)^{-1}$ was increased as the samples become dirtier, or the scattering lifetime, $\tau$, is decreased. This is understood to be due to the increase of DOS as mentioned above as a result of gap anisotropy smearing by impurity 
scatterings [5, 18]. We point out in this paper that this may also be understood in terms of impurity vertex correction (IVC). We found, as will be detailed below, that in the weak coupling limit the ratio of the coherence peak to normal state Korringar value roughly doubles as one goes from clean to dirty limit in rough agreement with experimental observations [5. 16. 17]. The enormous enhancement of NMR coherence peak reported in some organic superconductors, on the other hand, may be attributed to thermal fluctuations of magnetic flux lines [19].

It is well known that the IVC is very important in the transport properties of metals [20]. The scattering lifetime $\tau$ is changed to the transport lifetime $\tau_{t r}$ due to IVC, which appears in, for example, $\sigma(0)=n e^{2} \tau_{t r} / m$, where $n, e$ and $m$ are, respectively, the number density, charge and effective mass of the charge carriers. Because $T_{1}^{-1}$ and microwave conductivity, $\lim _{\omega \rightarrow 0} \sigma(\omega)$, have the same "plus coherence factor" [11], we may expect that the IVC may also be important for NMR relaxation rate. Maki and Fulde (MF) 21] studied this problem some 30 years ago assuming an infinite Fermi energy, $\epsilon_{F}$. They found the impurity vertex function $\widehat{\Gamma}(\omega, \vec{q}) \rightarrow 1$ in the limit of the momentum transfer $\vec{q} \rightarrow \infty$ and $\omega \rightarrow 0$, and concluded that $T_{1}^{-1}$, consequently, remains unrenormalized under IVC. This conclusion, however, is not rigorous because $T_{1}^{-1}$ is given by an integral over the momentum transfer $\vec{q}$, not by the limit $\vec{q} \rightarrow \infty$. As will be discussed later, $T_{1}^{-1}$ involves integrals over $\overrightarrow{k^{\prime}}$ and $\vec{q}$ of the product $\operatorname{Tr}\left[\widehat{G}\left(\overrightarrow{k^{\prime}}\right) \widehat{G}\left(\overrightarrow{k^{\prime}}+\vec{q}\right) \widehat{\Gamma}(\vec{q})\right]$, where $\operatorname{Tr}$ and $G$ stand for trace and Green's function, respectively. Since superconducting pairing occurs mainly within a narrow region around the Fermi surface, we can see that the main contribution to $T_{1}^{-1}$ comes from the region $\left|\overrightarrow{k^{\prime}}\right| \approx p_{F}$ and $\left|\overrightarrow{k^{\prime}}+\vec{q}\right| \approx p_{F}$, that is, from the region $Q=\left(q^{2}+2 \vec{q} \cdot \overrightarrow{k^{\prime}}\right) / 2 m \approx 0$, where $p_{F}$ is the Fermi momentum. The $Q \approx 0$ region and the $q \rightarrow \infty$ considered by MF should be distinguished. Moreover, the impurity vertex function which enters $T_{1}^{-1}$ has a singularity as $q \rightarrow 0$, which contributes to the $Q \approx 0$ region, as will be discussed. These observations call for a more careful analysis of the IVC effects on spin-lattice relaxation rate than the $q \rightarrow \infty$ of MF. In this paper, we report such a calculation. We find an explicit expression for $T_{1}^{-1}$ including IVC with a simple approximation about angular average which agrees with the 
approximate conclusion of $\mathrm{MF}$ that $T_{1}^{-1}$ remains unrenormalized under IVC for $\ell / \xi \gg 1$, where $\ell=v_{F} \tau$ is the electron mean free path and $\xi=\hbar v_{F} / \pi \Delta_{0}$ is the superconducting coherence length, $\Delta_{0}$ the gap function at zero temperature. As $\ell / \xi$ is reduced, the NMR coherence peak is found to increase due to IVC.

The nuclear spin-lattice relaxation rate $T_{1}^{-1}$ is given by 22

$$
\frac{1}{T_{1}}=\lim _{\omega \rightarrow 0} \frac{1}{1-e^{-\beta \omega}} \sum_{\vec{q}}\left|A_{q}\right|^{2} \operatorname{Im}\left[\chi_{+-}(\omega+i \delta, \vec{q})\right],
$$

where $A_{q}$ is a form factor related with the conduction electron wavefunctions, and $\chi_{+-}(\omega, \vec{q})$ is a spin-spin correlation function at frequency $\omega$ and momentum transfer $\vec{q}$, obtained through analytic continuation from the Matsubara function $\chi_{+_{-}}(i \omega, \vec{q})$. It should be calculated with non-magnetic impurities included fully self-consistently. We do this by renormalizing the quasi-particle Green's function due to impurity scattering in the $t$-matrix approximation and also by including the impurity vertex correction in ladder approximation, in accordance with the Ward identity [3,20]. This procedure results in the Bethe-Salpeter equation, which is to be solved for the vertex function. The renormalized $2 \times 2$ matrix single particle Matsubara Green's function due to impurity scattering is given by

$$
\widehat{G}\left(i p_{n}, \vec{k}\right)=-\frac{i \widetilde{W}_{n}+\widetilde{\xi}_{k} \tau_{3}+\widetilde{\phi}_{n} \tau_{1}}{\widetilde{W}_{n}^{2}+\widetilde{\xi}_{k}^{2}+\widetilde{\phi}_{n}^{2}}
$$

where $\tau_{i}$ 's are the Pauli matrices operating on the Nambu space. $i \widetilde{W}_{n}=i p_{n} \widetilde{Z}_{n}, \widetilde{\phi}_{n}=\widetilde{\Delta}_{n} \widetilde{Z}_{n}$, where $p_{n}=\pi(2 n+1) / \beta$ is the Matsubara frequency, and $\widetilde{Z}_{n}=\widetilde{Z}\left(i p_{n}\right)$ and $\widetilde{\Delta}_{n}=\widetilde{\Delta}\left(i p_{n}\right)$ are, when analytically continued to real frequency, the renormalization function and gap function, respectively. A tilde on a variable denotes that it is renormalized by impurity scatterings, and $\xi_{k}=k^{2} / 2 m-\mu$, where $\mu$ is the chemical potential. The self-energy, $\widehat{\Sigma}\left(i p_{n}, \vec{k}\right)$, due to impurity scatterings is then given by

$$
\widehat{G}^{-1}\left(i p_{n}, \vec{k}\right)=\widehat{G}_{0}^{-1}\left(i p_{n}, \vec{k}\right)-\widehat{\Sigma}\left(i p_{n}, \vec{k}\right), \quad \widehat{\Sigma}\left(i p_{n}, \vec{k}\right)=n_{i} \widehat{T}\left(i p_{n}, \vec{k}\right)
$$

as shown in Fig. 1(a), where $n_{i}$ is impurity concentration, $\widehat{T}$ the $t$-matrix for impurity scattering, and $\widehat{G}_{0}$ is the Green's function for pure superconductors. We treat the non- 
magnetic impurity scattering in a self-consistent $t$-matrix approximation as shown in Fig. 1(b) to obtain

$$
\widehat{T}=\frac{1}{\pi N_{F}} \frac{U}{1+U^{2}}\left[\tau_{3}-\frac{U}{\sqrt{\widetilde{W}_{n}^{2}+\widetilde{\phi}_{n}^{2}}}\left(i \widetilde{W}_{n}-\widetilde{\phi}_{n} \tau_{1}\right)\right] .
$$

Then there exists a simple scaling relation between pure $\left(i W_{n}, \phi_{n}\right)$ and impure $\left(i \widetilde{W}_{n}, \widetilde{\phi}_{n}\right)$ superconductivity [11, 14].

$$
\begin{aligned}
i \widetilde{W}_{n} & =\eta_{n} i W_{n}, \quad \widetilde{\phi}_{n}=\eta_{n} \phi_{n}, \quad \widetilde{\xi}=\xi+\frac{1}{2 \tau U}, \\
\eta_{n} & =1+\frac{1}{2 \tau \sqrt{W_{n}^{2}+\phi_{n}^{2}}}, \quad \frac{1}{2 \tau}=\frac{n_{i}}{\pi N_{F}} \frac{U^{2}}{1+U^{2}}=\frac{n_{i}}{\pi N_{F}} \sin ^{2} \delta_{N},
\end{aligned}
$$

where $n_{i}$ is impurity concentration and $\delta_{N}$ the phase shift due to impurity scattering, $U=$ $\pi N_{F} V=\tan \delta_{N}$, where $V$ is the impurity scattering potential energy.

The impurity vertex function, $\widehat{\Gamma}$, is calculated in ladder approximation as shown in Fig. 1(c). We have

$$
\widehat{\Gamma}\left(i p_{n}, i p_{m}, \vec{q}\right)=\tau_{0}+\sum_{\vec{k}^{\prime}} \widehat{T}\left(i p_{n}\right) \widehat{G}\left(i p_{n}, \vec{k}^{\prime}\right) \widehat{\Gamma}\left(i p_{n}, i p_{m}, \vec{q}\right) \widehat{G}\left(i p_{m}, \vec{k}^{\prime}+\vec{q}\right) \widehat{T}\left(i p_{m}\right)
$$

This is the Bethe-Salpeter equation at arbitrary momentum transfer $\vec{q}$ and frequency $i \omega_{m-n}=i p_{m}-i p_{n}=2 \pi(m-n) / \beta$ in ladder approximation, which we need to solve for $\widehat{\Gamma}$. Then $T_{1}^{-1}$ can be calculated from Eq. (11) and

$$
\begin{aligned}
\chi_{+-}(i \omega) & =\frac{1}{\beta} \sum_{i p} P(i p, i p+i \omega), \\
P(i p, i p+i \omega) & =\sum_{\overrightarrow{k^{\prime}}, \vec{q}} \operatorname{Tr}\left[\widehat{G}\left(i p+i \omega, \vec{k}^{\prime}+\vec{q}\right) \widehat{G}\left(i p, \vec{k}^{\prime}\right) \widehat{\Gamma}(i p, i p+i \omega, \vec{q})\right] .
\end{aligned}
$$

We will omit the subscripts of the Matsubara frequency for convenience. After the analytic continuation of $i \omega \rightarrow \omega+i \delta$ and the limit $\omega \rightarrow 0$, Eq. (7) is reduced to [20

$$
\lim _{\omega \rightarrow 0} \frac{1}{\omega} \operatorname{Im}\left[\chi_{+-}(\omega+i \delta)\right]=\int_{-\infty}^{\infty} \frac{d \epsilon}{2 \pi} \frac{\partial f(\epsilon)}{\partial \epsilon}\{P(\epsilon-i \delta, \epsilon+i \delta)-\operatorname{Re}[P(\epsilon+i \delta, \epsilon+i \delta)]\}
$$

where $f(\epsilon)=1 /\left(1+e^{\beta \epsilon}\right)$ is the Fermi distribution function. In order to solve Eq. (6), we employ the following factorization as with Hirschfeld et. al. 23]. 


$$
\begin{aligned}
L_{i j}(i p, i p+i \omega, \vec{q}) & =\frac{1}{2} \sum_{\overrightarrow{k^{\prime}}} \operatorname{Tr}\left[\tau_{i} \widehat{G}\left(i p, \vec{k}^{\prime}\right) \tau_{j} \widehat{G}\left(i p+i \omega, \vec{k}^{\prime}+\vec{q}\right)\right] \\
S_{i j}(i p, i p+i \omega) & =\frac{1}{2} \operatorname{Tr}\left[\tau_{i} \widehat{T}(i p) \tau_{j} \widehat{T}(i p+i \omega)\right] \\
\Gamma_{i}(i p, i p+i \omega, \vec{q}) & =\frac{1}{2} \operatorname{Tr}\left[\tau_{i} \widehat{\Gamma}\right] .
\end{aligned}
$$

Then Eq. (6) is reduced to

$$
\Gamma_{i}=\delta_{i, 0}+\sum_{k, l} S_{i k} L_{k l} \Gamma_{l}
$$

and Eq. (7) to

$$
\chi_{+-}(i \omega)=\sum_{i p, \vec{q}} \sum_{j} L_{0 j} \Gamma_{j} .
$$

The summations over $\overrightarrow{k^{\prime}}$ in Eq. (17) are carried out with a constant density of states, $\sum_{\overrightarrow{k^{\prime}}}=\int_{-\infty}^{\infty} N_{F} d \xi_{k^{\prime}} \frac{1}{2} \int_{-1}^{1} d \nu$, where $\nu=\cos (\theta), \theta$ is the angle between $\overrightarrow{k^{\prime}}$ and $\vec{q}$, and with the approximation $\xi_{\overrightarrow{k^{\prime}}+\vec{q}}=\left(\overrightarrow{k^{\prime}}+\vec{q}\right)^{2} / 2 m-\mu \approx \xi_{\overrightarrow{k^{\prime}}}+Q(\nu)$, where $Q(\nu)=\left(2 p_{F} q \nu+q^{2}\right) / 2 m$, which are standard approximations in the theory of superconductivity [11]. It is then straightforward to evaluate $L_{i j}$. For instance,

$$
\begin{aligned}
L_{00}(i p, i p+i \omega, q) & =N_{F} \frac{\pi \tau}{2}\left\langle f_{-}(i p, i p+i \omega, q)\right\rangle\left(-\phi_{n} \phi_{m}+W_{n} W_{m}-\sqrt{ }_{n} \sqrt{ }_{m}\right) / \sqrt{ }_{n} \sqrt{ }_{m} \\
\left\langle f_{ \pm}(i p, i p+i \omega, q)\right\rangle & =\frac{2 i}{\tau}\left[\left\langle\frac{1}{Q-i \sqrt{n}_{n}-i \sqrt{f}_{m}}\right\rangle \pm\left\langle\frac{1}{Q+i \mathcal{f}_{n}+i \sqrt{F}_{m}}\right\rangle\right] \\
& =\frac{i}{4 \tau \epsilon_{F} x}\left[\log \left(\frac{x^{2}+x-i \delta_{s}}{x^{2}-x-i \delta_{s}}\right) \pm \log \left(\frac{x^{2}+x+i \delta_{s}}{x^{2}-x+i \delta_{s}}\right)\right]
\end{aligned}
$$

where $\sqrt{ }_{n}=\sqrt{W_{n}^{2}+\phi_{n}^{2}}, \mathfrak{V}_{n}=\sqrt{\widetilde{W}_{n}^{2}+\widetilde{\phi}_{n}^{2}}, x=q /\left(2 p_{F}\right)$, and $\delta_{s}=\left(\mathfrak{F}_{n}+\mathfrak{V}_{m}\right) / \epsilon_{F}=$ $\left(\sqrt{ }_{n}+\sqrt{ }_{m}+1 / \tau\right) / \epsilon_{F}$ using the scaling relation of Eq. (司). \langle\rangle represents an angular average over $\nu$, that is, $\langle F\rangle=\frac{1}{2} \int_{-1}^{1} d \nu F(\nu)$. Note that Eq. (12) is expressed in terms of untilded variables taking advantage of Eq. (5) because its numerator and denominator are of the same powers in $\widetilde{W}$ and $\widetilde{\phi}$.

Let us first consider the Born limit of $\delta_{N} \rightarrow 0$. Then, $\widehat{T}=V \tau_{3}, \frac{1}{2 \tau}=n_{i} \pi N_{F} V^{2}$, and $S_{i j}$ of Eq. (9) becomes a diagonal matrix. In this case we can find an explicit solution $\Gamma(i \omega, \vec{q})$ to Eq. (10) for arbitrary $\vec{q}$ and $i \omega$. We will discuss the general case of finite $\delta_{N}$ later. After somewhat laborious calculations, we obtain 


$$
\begin{aligned}
\Gamma_{0}= & 1+\frac{\left\langle f_{-}\right\rangle+\left\langle f_{-}\right\rangle^{2}-\left\langle f_{+}\right\rangle^{2}}{\left(1+\left\langle f_{-}\right\rangle+\left\langle f_{+}\right\rangle\right)\left(1+\left\langle f_{-}\right\rangle-\left\langle f_{+}\right\rangle\right)} \frac{W_{n} W_{m}-\phi_{n} \phi_{m}-\sqrt{ }_{n} \sqrt{ }_{m}}{2 \sqrt{ }_{n} \sqrt{ }_{m}}, \\
\Gamma_{1}= & \frac{\left\langle f_{-}\right\rangle+\left\langle f_{-}\right\rangle^{2}-\left\langle f_{+}\right\rangle^{2}}{\left(1+\left\langle f_{-}\right\rangle+\left\langle f_{+}\right\rangle\right)\left(1+\left\langle f_{-}\right\rangle-\left\langle f_{+}\right\rangle\right)} \frac{i W_{n} \phi_{m}+i W_{m} \phi_{n}}{2 \sqrt{ }_{n} \sqrt{ }_{m}}, \\
\Gamma_{2}= & \frac{\left\langle f_{+}\right\rangle}{\left(1+\left\langle f_{-}\right\rangle+\left\langle f_{+}\right\rangle\right)\left(1+\left\langle f_{-}\right\rangle-\left\langle f_{+}\right\rangle\right)} \frac{W_{n} \sqrt{m}_{m}-W_{m} \sqrt{ }_{n}}{2 \sqrt{ }_{n} \sqrt{ }_{m}}, \\
\Gamma_{3}= & \frac{-\left\langle f_{+}\right\rangle}{\left(1+\left\langle f_{-}\right\rangle+\left\langle f_{+}\right\rangle\right)\left(1+\left\langle f_{-}\right\rangle-\left\langle f_{+}\right\rangle\right)} \frac{\phi_{n} i \sqrt{ }_{m}+\phi_{m} i \sqrt{ }_{n}}{2 \sqrt{ }_{n} \sqrt{ }_{m}} .
\end{aligned}
$$

We substitute this into Eq. (11) to finally obtain

$$
\begin{aligned}
\chi_{+-}(i \omega) & =\sum_{i p}\left[\frac{-\phi_{n} \phi_{m}+W_{n} W_{m}}{\sqrt{ }_{n} \sqrt{ }_{m}}-1\right] \Lambda(i p, i p+i \omega), \\
\Lambda(i p, i p+i \omega) & =\int_{0}^{\infty} d q q^{2} \frac{\left\langle f_{-}\right\rangle+\left\langle f_{-}\right\rangle^{2}+\left\langle f_{+}\right\rangle^{2}\left(W_{n} W_{m}+\phi_{n} \phi_{m}\right) /\left(2 \sqrt{n}_{n} \sqrt{m}_{m}\right)}{\left(1+\left\langle f_{-}\right\rangle+\left\langle f_{+}\right\rangle\right)\left(1+\left\langle f_{-}\right\rangle-\left\langle f_{+}\right\rangle\right)} .
\end{aligned}
$$

Note that in the limit $q$ (or $x) \rightarrow 0,\left\langle f_{-}(\epsilon-i \delta, \epsilon+i \delta)\right\rangle$ and $\left\langle f_{+}(\epsilon-i \delta, \epsilon+i \delta)\right\rangle$ of Eq. (13), which we need for analytic continuation as in Eq. (8), reduce to $-1+\frac{1}{3}\left(\epsilon_{F} \tau x\right)^{2}+\mathcal{O}\left(x^{4}\right)$ and $i \epsilon_{F} \tau x^{2}+\mathcal{O}\left(x^{4}\right)$, respectively. The vertex functions given by Eq. (14), consequently, diverge as $q \rightarrow 0$, which is canceled by the phase factor of $q^{2}$ in Eq. (15). We need to carry out the summation over the Matsubara frequency ip and the momentum $\vec{q}$ in Eq. (15), and then take analytic continuation and the limit $\omega \rightarrow 0$ as given in Eq. (8). Neglecting IVC amounts to taking $\Lambda=1$, for which we obtain the well known result [24]

$$
\begin{aligned}
\frac{1}{T_{1} T} & \propto \int_{0}^{\infty} d \epsilon \frac{\partial f}{\partial \epsilon}\left\{\left[\operatorname{Re}\left(\frac{\epsilon}{\sqrt{\epsilon^{2}-\Delta^{2}}}\right)\right]^{2}+\left[\operatorname{Re}\left(\frac{\Delta}{\sqrt{\epsilon^{2}-\Delta^{2}}}\right)\right]^{2}\right\} . \\
& \rightarrow \int_{\Delta}^{\infty} d \epsilon \frac{\partial f}{\partial \epsilon}\left\{\frac{\epsilon^{2}+\Delta^{2}}{\epsilon^{2}-\Delta^{2}}\right\} .
\end{aligned}
$$

Eq. (17) follows in the weak coupling limit. Eq. (15) may not be evaluated analytically, but is greatly simplified in the following approximation that treats the angular average differently:

$$
\Lambda \approx \int_{0}^{\infty} d q q^{2}\left\langle\frac{f_{-}+f_{-}^{2}+f_{+}^{2}\left(W_{n} W_{m}+\phi_{n} \phi_{m}\right) /\left(2 \sqrt{n}_{n} \sqrt{m}_{m}\right)}{\left(1+f_{-}+f_{+}\right)\left(1+f_{-}-f_{+}\right)}\right\rangle .
$$

With the approximation of Eq. (18), we find, in the weak coupling limit, after the analytic continuation of Eq. (8) 


$$
\frac{1}{T_{1} T} \propto \int_{\Delta}^{\infty} d \epsilon \frac{\partial f}{\partial \epsilon}\left\{\frac{\epsilon^{2}}{\epsilon^{2}-\Delta^{2}}+\frac{\Delta^{2}}{\epsilon^{2}-\Delta^{2}}\left[1+\frac{2}{1+(4 \tau)^{2}\left(\epsilon^{2}-\Delta^{2}\right)}\right]\right\}
$$

We also considered the case where the impurity potential is not weak, that is, where the phase shift $0<\delta_{N} \leq \pi / 2$. The resulting equation is extremely complicated. For $\omega \rightarrow 0$, however, we were able to find an explicit expression corresponding to Eq. (19) with the approximation of Eq. (18): Eq. (19) is still valid for arbitrary phase shift. All that is needed is that $\frac{1}{2 \tau}=n_{i} \pi N_{F} V^{2}$ of the Born limit should be extended to $\frac{1}{2 \tau}=\frac{n_{i}}{\pi N_{F}} \frac{U^{2}}{1+U^{2}}$ as given in Eq. (5). Eq. (19) reduces to Eq. (17) in the limit $\tau \rightarrow \infty$. This agrees with the previous result of $\mathrm{MF}$ that $1 / T_{1}$ remains unrenormalized under the IVC. In the limit $\tau \rightarrow 0$, on the other hand,

$$
\frac{1}{T_{1} T} \rightarrow \int_{\Delta}^{\infty} d \epsilon \frac{\partial f}{\partial \epsilon}\left\{\frac{\epsilon^{2}+3 \Delta^{2}}{\epsilon^{2}-\Delta^{2}}\right\}
$$

The dominant contribution to $1 /\left(T_{1} T\right)$ comes from the region $\epsilon \approx \Delta$. Consequently, comparing Eqs. (17) and (20), we can easily see that the ratio of coherence peak to normal state value is bigger in dirty limit than in clean limit by a factor of about 2. In Fig. 2, we show the normalized NMR relaxation rate, $\left(T_{1} T\right)_{n} /\left(T_{1} T\right)_{s}$, calculated from Eq. (19), as a function of temperature for several values of $\ell / \xi$. We took the electron-phonon coupling constant $\lambda=0.5$ and the phonon frequency $\omega_{p h}=0.1 \mathrm{eV}$ for representative values. The solid, dot-dot-dashed, dot-dashed, and dashed curves correspond to $\ell / \xi=100,10,1$, and 0.1 , respectively. The curves of $\ell / \xi=0.1$ and 100 are, respectively, almost indistinguishable from those of the dirty and clean limit. The NMR coherence peak is increased by a factor of 1.7 as expected, as $\ell / \xi$ is decreased from 100 to 0.1 , which is in fair agreement with the experimental observations on Al- and In-based alloys [5,16,17]. This observation raises an interesting possibility that the experimental observation of the NMR coherence peak increase may be understood in terms of impurity vertex correction rather than the gap anisotropy smearing. Experimental distinction between the two effects is highly desirable in this regard.

To summarize, we considered the effects of impurity vertex correction on nuclear spinlattice relaxation rate within the Eliashberg formalism. The relaxation rate was evaluated 
and found to agree in clean limit with the previous approximate conclusion of Maki and Fulde that $1 /\left(T_{1} T\right)$ remains unrenormalized under the impurity vertex correction. As the scattering lifetime is decreased, on the other hand, the coherence peak in $1 /\left(T_{1} T\right)$ was found to increase due to the impurity vertex correction. The nuclear spin-lattice relaxation data measured on conventional superconductors as impurity scattering rates are varied may also be understood in terms of impurity vertex correction rather than the gap anisotropy smearing effects induced by impurity scatterings.

H.Y.C. was supported by Korea Science and Engineering Foundation through Grant No. 931-0200-003-2 and through Center for Theoretical Physics, Seoul National University, and by the Ministry of Education through Grant No. BSRI-94-2428. 


\section{REFERENCES}

[1] Slichter, Theory of Nuclear Magnetic Resonance, 2nd ed., Springer-Verlag (1991).

[2] J. Bardeen, L. N. Cooper, and J. R. Schrieffer, Phys. Rev. 108, 1175 (1957).

[3] J. R. Schrieffer, Theory of Superconductivity, Addison-Wesley (1964).

[4] P. B. Allen and B. Mitrovic, in Solid State Physics Vol. 37, edited by F. Seitz et al., Academic Press (1982), p. 1.

[5] D. E. McLaughlin, in Solid State Physics Vol. 31, edited by H. Ehrenreich et al., Academic Press (1976), p. 1.

[6] D. Pines, Physica B 199 \& 200, 300 (1994); P. Monthoux and D. Pines, Phys. Rev. B 47, 6069 (1993).

[7] N. Bulut and D. J. Scalapino, Phys. Rev. Lett. 68, 706 (1992).

[8] P. B. Allen and D. Rainer, Nature 349, 396 (1991).

[9] Y. O. Nakamura et. al, Solid State Commun. 86, 627 (1993).

[10] R. Akis and J. P. Carbotte, Solid State Commun. 78, 393 (1991).

[11] K. Maki, in Superconductivity, edited by R. D. Parks, Dekker (1967), p. 1035.

[12] H. Hasegawa, J. Phys. Soc. Jpn. 42, 779 (1977).

[13] H. Monien et al., Phys. Rev. B 43, 258 (1991); ibid., 275 (1991).

[14] A. A. Abrikosov, L. P. Gorkov, and I. E. Dzyloshinski, Methods of Quantum Field Theory in Statistical Physics, Dover (1965), chap. 7.

[15] P. W. Anderson, J. Phys. Chem. Solid 11, 26 (1959).

[16] Y. Masuda, Phys. Rev. 126, 1271 (1962).

[17] J. D. Williamson and D. E. MacLaughlin, Phys. Rev. B 8, 125 (1973). 
[18] J. R. Clem, Phys. Rev. 148, 392 (1966).

[19] L. Xing and Y. C. Chang, Phys. Rev. Lett. 73, 488 (1994).

[20] G. D. Mahan, Many-Particle Physics, 2nd ed., Plenum (1990), chap. 7.

[21] K. Maki and P. Fulde, Phys. Rev 140, A1586 (1965).

[22] T. Moriya, J. Phys. Soc. Jpn. 18, 516 (1963).

[23] P. Hirschfeld et al., Phys. Rev. B 37, 83 (1989).

[24] M. Fibich, Phys. Rev. Lett. 14, 561 (1965); ibid., 621 (1965). 


\section{FIGURES}

FIG. 1. Feynmann diagrams for (a) self-energy, $\widehat{\Sigma}$, (b) $t$-matrix for impufity scatterings, $\widehat{T}$, and (c) impurity vertex function, $\widehat{\Gamma}$. The solid double and single lines, respectively, stand for the renormalized, $\widehat{G}$, and bare Green's functions, $\widehat{G}_{0}$. The dashed double and single lines, respectively, stand for the $t$-matrix, $\widehat{T}$, and bare impurity scatterings, $V \tau_{3}$, and the shaded triagle in (c) represents the impurity vertex function, $\widehat{\Gamma}$.

FIG. 2. The normalized NMR relaxation rate, $\left(T_{1} T\right)_{n} /\left(T_{1} T\right)_{s}$, as a function of $T / T_{c}$. The solid, dot-dot-dashed, dot-dashed, and dashed lines were, respectively, calculated for $\ell / \xi=100,10,1$, and 0.1 with $\lambda=0.5$ and $\omega_{p h}=0.1 \mathrm{eV}$. 


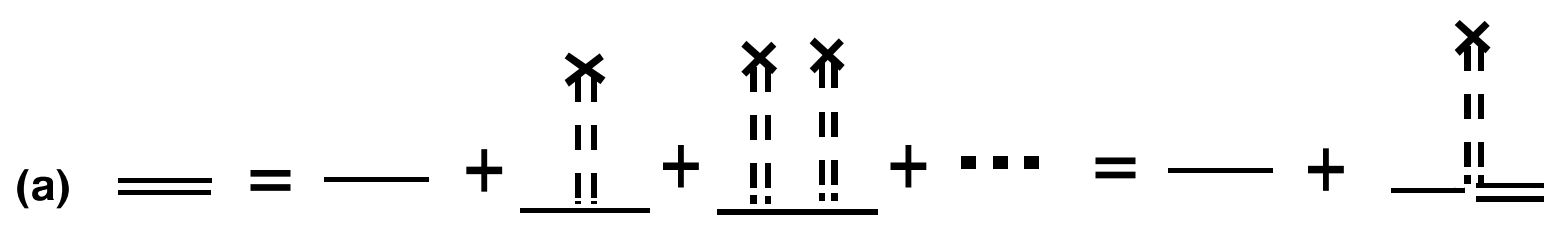

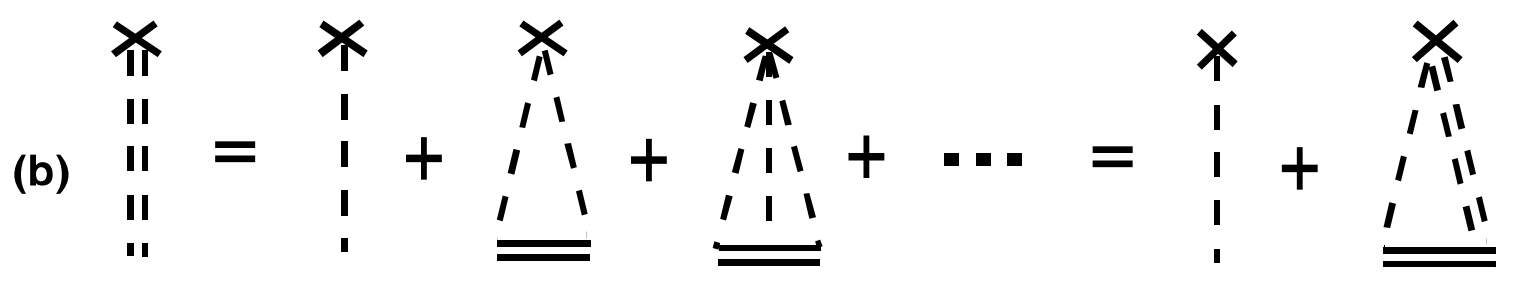

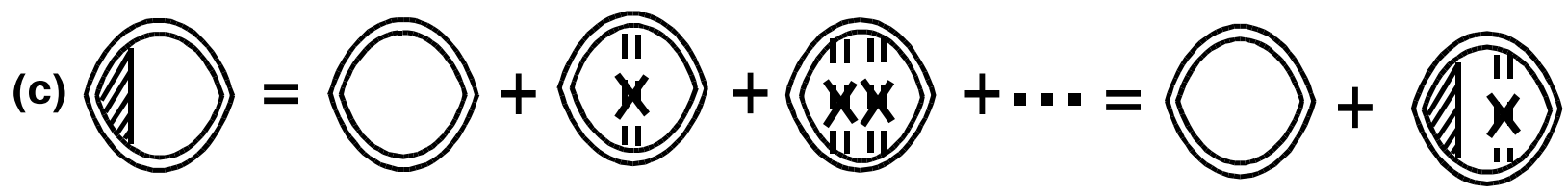

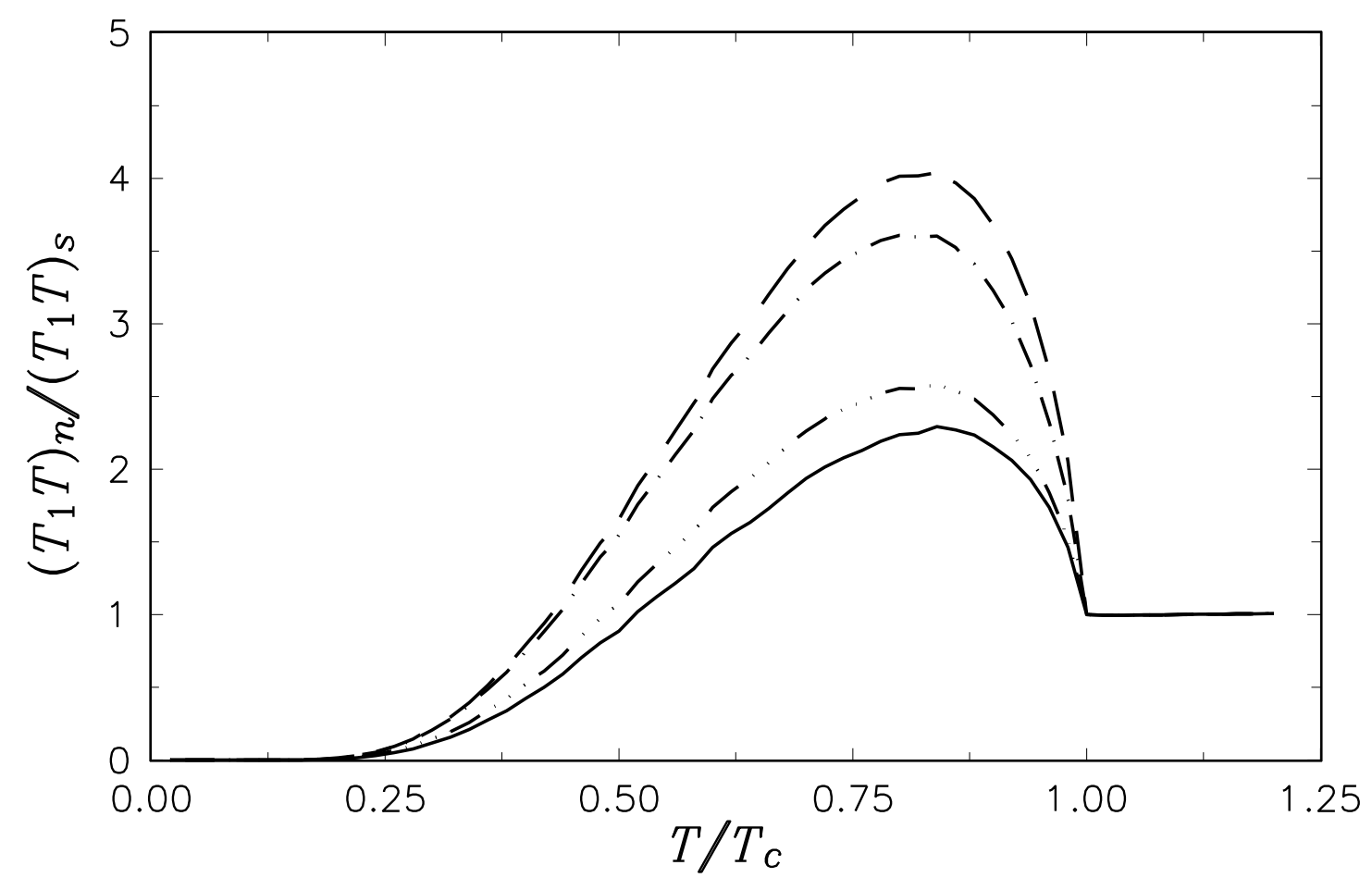

